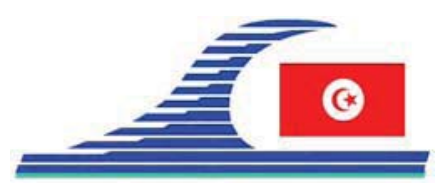

Conférence Méditerranéenne Côtière et Maritime

EDITION 1, HAMMAMET, TUNISIE (2009)

Coastal and Maritime Mediterranean Conference

Disponible en ligne - http://www.paralia.fr-Available online

\title{
Impact des activités anthropiques sur la qualité des sédiments du littoral de Rades Sud-Est (Tunis)
}

\author{
Hassen OUELHAZI ${ }^{1}$, Rachida TALBI ${ }^{1}$, Malika METHAMEM ${ }^{1}$, \\ Abedelkrim CHAREF ${ }^{1}$
}

1. Laboratoire des Géoressources, Centre de Recherches et des Technologies des Eaux (CERTE), Borj Cedria BP 273 Soliman 8020 Tunisie.

Abdelkrim.Charef@certe.rnrt.tn ; Malika.Methammam@certe.rnrt.tn ;

ouelhazihassen@yahoo.fr

\section{Résumé :}

En Tunisie, le golfe de Tunis qui est une zone semi fermée commence à manifester des signes de dégradation. Le long de l'oued Méliane, principal oued de la région et ses affluents. Le débit le long de l'oued est faible et ponctuel sauf en cas de pluviométries importantes ou de crues. Les S.T.E.P localisées dans la région sont la station de prétraitement à El Fahs qui déverse dans l'oued et la grande station de Rades ben Arous déversant dans la mer. Les dégâts environnementaux qui commencent à être de plus en plus visibles et saillants dans les parties côtières montrent l'importance d'étudier les rejets liquides et solides. Cette étude met l'accent sur l'influence des activités anthropiques sur un tronçon d'environ 2,5 km linéaires par une étude granulométrique, minéralogique et géochimique des sédiments de la plage entre Rades-Ezzahra. Ces polluants sont essentiellement drainés par les eaux et les sédiments de l'Oued Méliane et son affluent Oued Maezete. Les résultats montrent que cette plage est polluée par le $\mathrm{Cd}$ et la matière organique qui sont en partie d'origine anthropique. Cette pollution n'est pas très ancienne et la situation globale s'est améliorée par rapport à 1993.

\section{Mots-clés :}

Environnement côtier - Sédiments - Pollution minérale - Pollution organique (COT, extrait lipidique)

\section{Introduction}

Autour du bassin méditerranéen et suite à l'accroissement démographique et le développement des activités industrielles et agricoles, il y a eu une augmentation importante des quantités des rejets solides et liquides. Pour la plupart des cas, la mer méditerranéenne est le principal exutoire de ces rejets. Ces polluants minéraux et organiques ont parfois provoqué des dégâts apparents.

En Tunisie, plusieurs oueds déversent leur contenu pollué en mer. Dans le cas où elle est ouverte l'impact de ces produits est peu sensible. Mais lorsque la zone est fermée et/ou semi ouverte et fragile tel que le cas du Golfe de Tunis, la dégradation du milieu DOI: $10.5150 / \mathrm{cmcm} .2009 .054-1$ 
pourra être importante. Ainsi, les rejets pollués doivent être contrôlés pour évaluer leur impact sur cette zone sensible. L'oued Méliane avec son affluent l'oued Maezate est le principal fleuve qui alimente le petit golfe de Tunis. Il draine le versant nord oriental de la dorsale tunisienne et débouche dans la zone Ezzahra-Rades. Le contenu de cet oued est visiblement pollué et son impact sur cette frange du littoral ne laisse aucun doute. Ainsi, pour évaluer l'impact de ce cours d'eau sur cette partie côtière, on est intéressé à la zone la plus dégradée qui comprend la plage de Rades-Ezzahra, sur environ 2,5 km de long et $500 \mathrm{~m}$ de distance à la côte et de $3 \mathrm{~m}$ de bathymétrie.

Puisque les sédiments constituent un réceptacle des polluants particulaires, polluants solides et produits de précipitation et de compléxation, on évaluera les sédiments de cette zone.

\section{Matériel et méthode}

Pour bien répartir nos prélèvements, on a échantillonné 17 échantillons le long de cinq radiales orientée NE-SW. Pour avoir une idée sur l'évolution verticale des paramètres étudiés on a fait deux carottes de, 50 et $70 \mathrm{~cm}$. On a fait les analyses granulométriques et minéralogiques. On a dosé les métaux lourds de la fraction inférieure à $63 \mu \mathrm{m}$. On a déterminé le COT par Rock-Eval (II). On a réalisé l'extraction lipidique et les analyses de la fraction des hydrocarbures saturés et insaturés non aromatiques par chromatographie en phase liquide et gazeuse.

\section{Résultats et discussion}

\subsection{Les minéraux non argileux et argileux}

L'étude minéralogique implique que la fraction minérale non argileuse est dominée par la silice et les feldspaths. Tandis que les argiles sont dominées par la kaolinite et l'illite. Ces données montrent que les sédiments marins sont en partie d'origine détritique terrigène ce qui implique que l'oued Méliane et son affluent a drainé probablement avec ces minéraux des polluants.

L'étude granulométrique montre que les sédiments sont modérément classés à bien classés. Ainsi, dans cette zone du littoral l'hydrodynamisme des vagues a induit un classement des sédiments et aussi une redistribution des polluants des cours d'eau.

\subsection{Caractéristiques géochimiques de sédiments}

Les teneurs en cuivre, cadmium, en manganèse et en nickel sont respectivement de 0,9 à 43 ppm, de 0,2 à 2,2 ppm, de 246 à 454 ppm et de 30 à 75 ppm.

Pour évaluer le degré de dégradation de cette partie de la côte, on a calculé le facteur d'enrichissement; On a aussi comparé nos résultats aux données de ZEGGAF (1993) et RAIS (1999) (Tableau 1). Le facteur d'enrichissement $(r)$ et l'indicateur total $(R)$ sont définis par les équations suivantes (ADAMI et al., 2000) : 
$r=\frac{C_{\text {sed }}-C_{b a c k}}{C_{b a c k}}$, et : $R=\frac{\left(\sum_{1}^{n} r_{i}\right)}{n}$

$C_{\text {sed }}=$ est la concentration du métal dans les sédiments ; $C_{b a c k}=$ est la concentration du sédiment de référence non pollué ; $n=$ nombre de métaux. Selon la valeur de $r$ on a : $r<2$ zone non polluée, $2<r<3$ zone modérément polluée, $r>3$ zone très polluée.

Tableau 1. Facteurs d'enrichissement du cadmium, du manganèse, du cuivre et du nickel.

\begin{tabular}{|c|c|c|c|c|c|}
\hline Secteur & $r_{C d}$ & $r_{M n}$ & $r_{C u}$ & $r_{N i}$ & auteurs \\
\hline Petit Golfe de Tunis & $113 / 484$ & $-0,8 / 0,77$ & 0,14 & & $Z A G G A F, 1993$ \\
\hline $\begin{array}{l}\text { Littoral du Golfe de } \\
\text { Tunis }\end{array}$ & $27 / 199$ & $-0,34 / 0,57$ & $-0,79 / 1,05$ & $-0,28 / 0,73$ & RAIS, 1999 \\
\hline $\begin{array}{l}S \text {-W de petit Golfe de } \\
\text { Tunis }\end{array}$ & $6 / 63$ & $-0,29 / 0,3$ & $-0,97 / 0,13$ & $-0,22 / 0,8$ & $\begin{array}{l}\text { Cette étude, } \\
2007\end{array}$ \\
\hline
\end{tabular}

Le $r$ indique qu'en 2007, le Golfe de Tunis n'est pas pollué en Ni, Mn et $\mathrm{Cu}$ alors qu'il est très pollué en Cd mais la situation est améliorée par rapport à 1993 et 1999.

Les résultats montrent que les sédiments de deux carottes sont pollués par le cadmium et les sédiments de la base de la carotte sont moins pollués que ceux de la surface. L'ensemble des données montre que (a) le golfe de Tunis est pollué seulement par le Cd (b) cette pollution est récente (c) la situation s'est améliorée par rapport à 1993. On suggère que la source de pollution probable est anthropique.

\subsection{Teneurs en carbone organique total (COT)}

Pour les sédiments de surface, les concentrations sont élevées à l'embouchure d'oued Méliane (4,24\%) et d'oued Maezate (4,98\%) et sont largement en dessus des concentrations des sédiments marins. A priori, la matière organique est d'origine allochtone et drainée avec les sédiments et associée à la fraction vaseuse.

Les sédiments des sommets des carottes sont plus riches en matière organique. Ce résultat montre comme pour les métaux lourds que la pollution est récente. Cette pollution est drainée par les eaux de surface et est probablement d'origine anthropique. Les diagrammes COT-teneurs en métaux lourds montrent qu'on n'a pas de corrélation entre le COT et le Mn, Ni et Cd. Alors que le $r$ de COT-Cu est de 0,94. Par conséquent le $\mathrm{Cd}$ de notre zone d'étude et la matière organique n'ont peut-être pas la même origine. 


\subsection{Détermination de l'extrait lipidique}

Pour déterminer l'origine de la matière organique et de son degré d'évolution, on a dosé les teneurs en extrait chloroformique qui varient de 0,07 à $2 \%$ du sédiment. On constate que les zones les plus riches en extrait chloroformique se situent tout prés du bouchon vaseux d'oued Méliane. La distribution bimodale des n-alcanes ainsi que l'existence de légère imparité atteste de l'origine essentiellement biologique de la matière organique (BLUMER et al., 1971) vraisemblablement mixte végétale et marine. Ainsi les unresolved complex mixtures (UCM) constituent des signes de la biodégradation de la matière organique.

\section{Conclusions}

L'étude granulométrique, minéralogique et géochimique des sédiments montre que :

- l'oued Méliane et son affluent ont drainé probablement avec leurs minéraux les différents polluants,

- le golfe de Tunis est pollué seulement par le $\mathrm{Cd}$ et la matière organique (origine continentale probablement anthropique et marine) mais ces deux polluants n'ont pas la même origine. Cette pollution n'est pas très ancienne et la situation globale est améliorée, par rapport à 1993,

- cette pollution minérale et organique est d'origine anthropique. Les polluants sont drainés dans l'oued Méliane et son affluent avec les sédiments.

\section{Références bibliographiques}

ADAMI G., BARBERI G., RESENHOFER E. (2000). An improved index for monitoring metal pollutants in surface sediments Toxical. Environ. Chem. 77 (2000), pp 189-197.

BLUMER M., GUILLARD R.R.L., CHASE T. (1971). Hydrocarbons of Marine Phytoplankton. Marine Biology. Vol. 8, pp.183-189.

RAIS M. (1999). Géochimie des métaux lourds (Fe, $\mathrm{Mn}, \mathrm{Pb}, \mathrm{Cu}$ et $\mathrm{Cd}$ ) dans les eaux et les sédiments du littoral du Golfe de Tunis. Mobilité et impact des activités anthropiques. Thèse, Université de Tunis II, 211 p.

ZEGGAF M. (1993). Etude de l'impact des ouvrages de protection sur l'environnement du littoral du petit golfe de Tunis. DEA, Université de Tunis II, 79 p. 